\title{
DER ATLAS DER HYGIENE DES SÄUGLINGS UND KLEINKINDES ALS MASSNAHME ZUR VOLKSAUFKLÄRUNG
}

\author{
VON SIGRID STOCKEL
}

Berlin

Der «Atlas der Hygiene des Säuglings und Kleinkindes» gehört zu den Maßnahmen, mit denen am Ende des Kaiserreiches das Problem einer hohen Säuglingssterblichkeit bekämpft werden sollte. Die Säuglingssterblichkeit wurde seit den 90er Jahren von Ärzten, Nationalökonomen und Bevölkerungspolitikern in dem Bemühen diskutiert, die im internationalen Vergleich schlechte Stellung Deutschlands zu verbessern. Anfang des 20. Jhs starben von 100 Lebendgeborenen in Deutschland noch über 20 - ein Wert, der nur von Österreich und Ungarn übertroffen wurde, wo mehr als $21 \%$ lebendgeborene Säuglinge starben. In Frankreich und den Niederlanden lag die Säuglingssterblichkeit nur bei $14 \%$ und in Norwegen sogar bei weniger als $8 \%{ }^{1}$.

Am Ende des Ersten Weltkriegs war die Besorgnis um die Bevölkerungsentwicklung noch größer. Der Krieg hatte nicht nur hohe Verluste an Soldaten gefordert, sondern auch zu einem erheblichen Geburtenausfall geführt, der auf 3,6 Millionen geschätzt wurde². Die Geburtenziffer war bereits seit dem Beginn des Jahrhunderts gesunken: 1891 hatte es im Deutschen Reich 36 Geburten auf 1.000 Einwohner gegeben, vor dem Krieg nur noch $27^{3}$. 1918 betrug sie kriegsbedingt lediglich $14 \%$ ! Obwohl sich die Säuglingssterblich-

1 Festschrift zur Eröffnung des Kaiserin Auguste Victoria-Hauses zur Bekämpfung der Säuglinssterblichkeit im Deutschen Reiche, Berlin 1909, S. 9-11.

2 Elster, Monatsschrift für öffentliche Gesundheitspflege 1922, H. 1/2.

3 In Berlin war der Rückgang noch deutlicher: 1891 lag die Geburtenziffer bei 27 Geburten je 1.000 Einwohner, $1912 / 13$ bei 20 . 
keit während des Krieges nicht erhöht hatte, lag sie im internationalen Vergleich mit 14,5\% noch wesentlich über den Werten in Frankreich und England, ganz zu schweigen von den skandinavischen Ländern ${ }^{4}$. Um eine halbwegs positive Bevölkerungsbilanz zu erzielen, waren also Maßnahmen zur Senkung der Säuglingssterblichkeit unbedingt erforderlich.

Der Zusammenhang zwischen sozialer Lage und einer hohen Sterblichkeit der Kleinkinder lag auf der Hand. Sozial engagierte Ârzte hatten den Aufbau eines Mutter- und Säuglingsschutzes gefordert und für dje Kriegszeit in Ansätzen durchgesetzt. Neben die Kritik an den sozialen Umständen trat jedoch zunehmend die an den traditionellen Pflegepraktiken der Mütter, die den neueren medizinischen Erkenntnissen widersprachen. Diese zu verändern, war ein vordringliches $\mathrm{Ziel}$, und es konnte nur erreicht werden durch Aufklärung über die Zusammenhänge von Umwelt und Krankheit und der Erziehung der Mütter zur Hygiene.

In diesem Kontext steht der Atlas, der genau dies mit seinen Bildtafeln versucht. Im folgenden möchte ich die Organisationen vorstellen, die den Atlas entwickelt haben, und anhand einiger Tafeln sein didaktisches Konzept verdeutlichen.

Die Bekämpfung der Säuglingssterblichkeit wurde erst in der Weimarer Republik in die gesetzgeberische Verantwortung des Staates übernommen. 1918 gehörte sie noch in den Bereich privater Wohltätigkeit sowie der kommunalen Fürsorge. Dieser Umstand wird auch am Atlas selbst deutlich. Er wurde herausgegeben

- mit Unterstützung des Hauptvorstandes des Vaterländischen Frauenvereins,

- von Prof. Dr. Leo Langstein, Direktor des Kaiserin Auguste Victoria-Hauses zur Bekämpfung der Säuglingssterblichkeit im Deutschen Reich,

- und Dr. Fritz Rott, Direktor des Organisationsamtes für Säuglingsschutz der Kaiserin Auguste VictoriaHaus-Stiftung.

Hiermit sind die drei wichtigsten überregionalen Organisationen zur Bekämpfung der Säuglingssterblichkeit im

4 1920/24, vgl. Handbuch der europäischen Wirtschafts- und Sozialgeschichte, hrsg. v. W. Fischer u. a., Bd 6, Stuttgart 1988, S. 33: Sowjetunion 17,8\%; Osterreich 14,2\%; Deutschland 12,7\%; Frankreich $10,5 \%$; Großbritannien $7,7 \%$; Schweden $6,1 \%$; Norwegen $5,3 \%$. 
Kaiserreich genannt. Der «Vaterländische Frauenverein» war ein privater patriotischer Wohltätigkeitsverein, der innerhalb des Spektrums der privaten Wohltätigkeit am professionellsten organisiert war. In mehreren deutschen Gro $\beta$ städten hatten Ortsverbände des Vaterländischen Frauenvereins Säuglingsfürsorgestellen eingerichtet ${ }^{5}$, in denen Arbeiterfrauen ihre Kinder einem Arzt vorstellen konnten (Abb. 1).

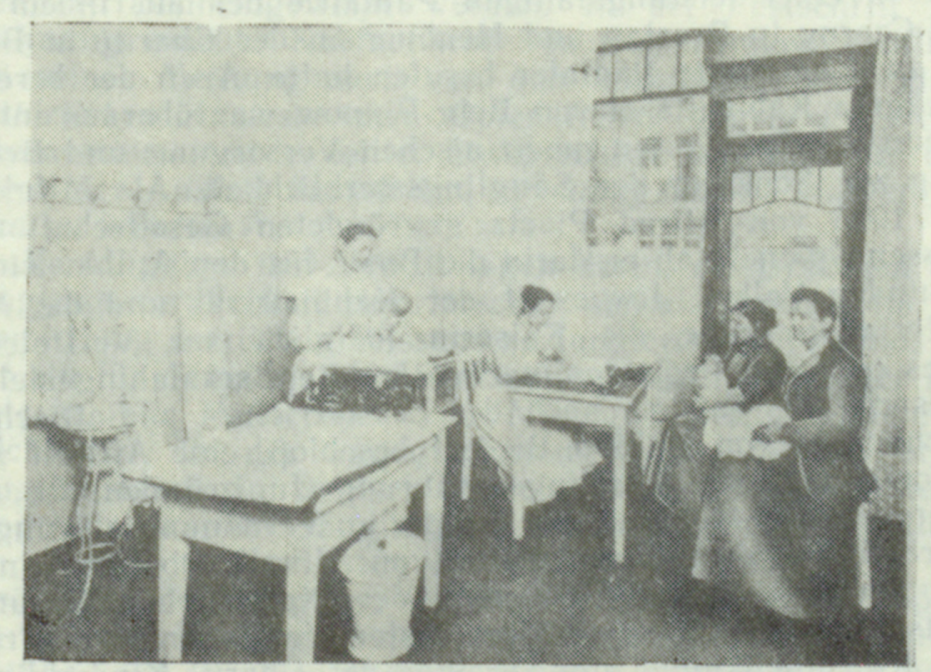

Abb. 1: Säuglingsfürsorgestelle

Das Kind wurde gewogen, seine körperliche Entwicklung festgestellt, und der Mutter wurden entsprechende Pflegehinweise gegeben. Vor allem aber wurde sie zum Stillen ermutigt. In einigen Städten besuchten ausgebildete Fürsorgeschwestern die Mütter zuhause, um die Pflege der Kinder zu überwachen.

Das Kaiserin Auguste Victoria-Haus, nach seiner Stifterin benannt, war als Reichsanstalt zur Bekämpfung der Säuglingssterblichkeit eingerichtet worden, um die Bedin-

5 Der Verein hatte außerdem auf Initiative der Kaiserin hin ein Merkblatt zur Säuglingspflege erstellt, das von den Standesbeamten verteilt wurde. 
gungen der Säuglingssterblichkeit zu erforschen und Mittel und Wege zu ihrer Senkung zu entwickeln. Die Kaiserin selbst Mutter von acht Kinder und außerdem patriotisch besorgt - hatte 1905 ihren Kabinettsrat Dr. Carl von BehrPinnow beauftragt, mit geeigneten Personen über ein gezieltes Vorgehen zu beraten. Bereits vorher hatte der Ministerialdirektor im Ministerium für geistliche, Unterrichts- und Medizinalangelegenheiten, Friedrich Althoff, sich um den Aufbau einer leistungsfähigen Pädiatrie bemüht, indem er mit Czerny in Breslau und Heubner an der Charité in Berlin zwei berühmte Pädiater berufen hatte. Auch der bereits erwähnte Kabinettsrat von Behr-Pinnow war überaus interessiert an einer guten medizinischen Versorgung und Beratung zur Senkung der Säuglingssterblichkeit. Als Mitglied der 1905 von Alfred Ploetz gegründeten Gesellschaft für Rassenhygiene ${ }^{6}$ sah er darin die Basis für den Aufbau eines gesunden Volkes. Inwieweit der Kabinettsrat und die Ministerialbürokratie die Kaiserin lediglich zur Vertreterin ihres eignenen Projektes machen konnten, sei dahingestellt? Tatsache ist, da $\beta$ sie nach der Lektüre eines pädiatrischen Fachbuches von Philipp Biedert beschlo $\beta$, eine Anstalt zur Erforschung der Säuglingsernährung zu gründen. da die überwiegende Mehrzahl der Kinder an Verdauungsstörungen starb. Diese Vorstellung wurde von Althoff, Obermedizinalrat Dietrich, dem Präsidenten des Kaiserlichen Gesundheitsamtes Bumm sowie dem ersten deutschen Ordinarius für Kinderheilkunde und Direktor der Charité-Kinderklinik, Otto Heubner, prinzipiell geteilt. Mehrere Mitglieder des Finanzministeriums sowie des Ministeriums für geistliche, Unterrichts- und Medizinalangelegenheiten, die bekannten Pädiater sowie patriotisch engagierte Persönlichkeiten aus den preußischen Provinzen und den deutschen Ländern schlossen sich 1906 im Reichstag zu einem «Deutschen Komitee zur Errichtung einer Musteransalt zur Bekämpfung der Säuglingssterblichkeit» zusammen. Die Kaiserin über-

6. Labisch, Alfons/Tennstedt, Florian: Der Weg zum «Gesetz über die Vereinheitlichung des Gesundheitswesens» vom 3. Juli 1934. Entwicklungslinien und -momente des staatlichen und kommunalen Gesundheitswesen in Deutschland, Teil 1 (=Schriftenreihe der Akademie für öffentliches Gesundheitswesen in Düsseldorf, Bd. 13,1), Düsseldorf 1985 , S. 155.

7 Säuglingspalast mit Kuhstall. Das Kaiserin Auguste Victoria-Haus war die Wiege der sozialen Pädiatrie, in: FU-Info 8-9/89, S. 2-4, hier S. 2. 
nahm das Protektorat, stiftete 25.000 Reichsmark und warb um weitere Mittel, die aus allen Landesteilen eingingen und Anfang 1907 eine Summe von 950.000 Goldmark erreichten ${ }^{8}$. Die Stadt Charlottenburg schenkte zur Silberhochzeit des Kaiserpaares ein an den Schloßpark angrenzendes Grundstück, der Kaiser verpflichtete seine Lieblingsarchitekten Ludwig Hofímann und Alfred Messel zu einem repräsentativen Bauplan, und bereits 1909 konnte das Kaiserin Auguste Victoria-Haus eingeweiht werden. In der Festschrift zur Eröfinung machte Kabinettsrat von Behr-Pinnow deutlich, da $\beta$ seiner Meinung nach von den Säuglingen viele einer «natürlichen Auslese» zum Opfer fielen, gegen die nichts unternommen werde sollte. Entsprechend schränkte er den Zweck der Anstalt darauf ein, «gesundgeborene Kinder gesund $z u$ erhalten $»^{9}$.

Die Aufgaben der Anstalt lagen darin, die Ursachen der Säuglingssterblichkeit zu erforschen und als Zentrale für die Vermittlung ärztlichen Wissens zu fungieren. Angeschlossen war ein für damalige Verhältnisse ungewöhnlich großer Labortrakt, ein Musterstall zur Milchgewinnung und eine Milchküche. In der angegliederten Klinik, die als erste Säuglingsklinik in Deutschland eine Entbindungsabteilung integrierte, wurden Säuglinge und wenig später auch ältere Kinder nach den neuesten Kenntnissen behandelt. Für die Ernährung der Jüngsten waren Ammen angestellt. Außerdem wurden Fürsorgerinnen und Säuglingsschwestern ausgebildet, und in einer angegliederten Fürsorgestelle Kinder ambulant betreut.

Das Haus wurde bis 1911 von dem Pädiater Arthur Keller geleitet, der sich bei Czerny in Breslau habilitiert und mit ihm zusammen ein zweibändiges Lehrbuch über Ernährung und Ernährungsstörungen des Kindes verfasst hatte ${ }^{10}$. Sein Stellvertreter und baldiger Nachfolger war Langstein,

8 Festschrift vgl. Anm. 1, S. 20. Stürzbecher, Manfred: die Vor-, Grün-

- dungs- und Frühgeschichte des Kaiserin Auguste Victoria-Hauses (KAVH) zur Bekämpfung der Säuglingssterblichkeit im Deutschen Reiche im Spiegel der Reichstagsdebatten 1900-1930, in: Schriftenreihe zur Geschichte der Kinderheilkunde aus dem Archiv des KAVH. Berlin, hrsg. van Leonore Ballowitz, Heft 4, 1987, S. 24-41, hier S. 25.

9 Festchrift vgl. Anm. 1, S. 13.

10 Keller, Arthur, in: Biographisches Lexikon hervorragender Arzte der letzten 50 Jahre, Berlin 1932, sowie Stürzbecher, Manfred: Keller, Arthur, in: NDB XI, Berlin 1977. 
der Schüler des Direktors der Charité-Kinderklinik, Otto Heubner, war.

In einem speziell eingerichteten Organisationsamt wurden Informationen über alle Maßnahmen gesammelt und Merkblätter zur Säuglingspflege erstelit, die durch ganz Deutschland verschickt wurden ${ }^{11}$. Direktor des Organisationsamtes war der Säuglingsarzt Fritz Rott, ebenso wie Langstein Heubner-Schüler ${ }^{12}$.

Die Reichsanstalt war geplant als ideale Verbindung von Wissenschaft und Praxis, wobei Praxis nicht nur Klinik bedeutete, sondern auch die Fürsorgetätigkeit, über die den Müttern Wissen über richtige Pflege und Ernährung des Säuglings vermittelt werden sollte ${ }^{13}$. Arvo Ylppö, der mehrere Jahre lang Arzt im Kaiserin Auguste Victoria-Haus und ein überzeugter Vertreter dieses Konzepts war, hat es dem Aufbau von Fürsorgestellen in Finnland zugrundegelegt ${ }^{14}$.

Zur Unterstützung der Wissensvermittlung war im Haus von Anfang an ein Museum vorgesehen. Die Idee war 1906 durch die Ausstellung für Säuglingspflege in Berlin angeregt worden, die ausgesprochen gut besucht worden war und die Möglichkeit und Notwendigkeit der Volksaufklärung durch dieses Medium verdeutlicht hatte ${ }^{15}$.

Bei allen Bemühungen um Aufklärung war klar, «da $\beta$ das geschriebene Wort niemals jene durchgreifende Kraft besitzen kann, die wir zur Erreichung unseres Zieles, Durchdringung des Volkes mit gründlichen Kenntnissen auf diesem wichtigen Gebiet der Gesundheitspflege, benötigen.

11 Arthur Keller, Aufgaben des KAVH, Organisation des Betriebes, in: Festschrift, S. 144-149.

12 Stürzbecher, Manfred: Beitrag zur Biographie von Fritz Rott (1878-1959), Begründer und Leiter des Organisationsamtes und zeitweiliger Mitdirektor des KAVH, in: Schriftenreihe zur Geschichte der Kinderheilkunde aus dem Archiv des Kaiserin Auguste VictoriaHauses (KAVH) - Berlin, hrsg. von Leonore Ballowitz, Heft 7, 1990, S. $29-44$.

13 Festschrift vgl. Anm. 1, S. 29.

14 Ylppö, Arvo: Mein Leben unter Kleinen und Großen, Lübeck 1987.

15 Rott, Fritz: Das Museum für Säuglingskunde, in: Bericht des Kaiserin Auguste Victoria-Hauses, Reichsanstalt zur Bekämpfung der Säuglings- und Kleinkindersterblichkeit, erstattet von Prof. Dr. L. Langstein vom 1.4. 1914 bis 31.3. 1915, S. $48-64$, hier S. 48 ff. 
Anschauungsunterricht und praktische Arbeit sind es, die dem geschriebenen und gesprochenem Wort den lebendigen Hintergrund geben ${ }^{16}$. Aus diesem Grund wurde am 4. Juni 1914, genau fünf Jahre nach Einweihung des Kaiserin Auguste Victoria-Hauses, ein Museum für Säuglingskunde eingerichtet. Mit großer Wahrscheinlichkeit hat die HygieneAusstellung von 1911 in Dresden anregend gewirkt, sie wird jedoch von den Säuglingsärzten nicht genannt, wogegen auf die Ausstellung zur Säuglingspflege von 1906 mehrfach hingewiesen wird.

Einzelne Stücke der Ausstellung von 1906 hatte das Kaiserin Auguste Victoria-Haus sofort für eine ständige Präsentation übernommen, die meisten Exponate wurden jedoch mithilfe einer großzügigen privaten Spende 1913 neu hergestellt. Die über 2.750 Gegenstände waren auf $500 \mathrm{qm}$ in zehn systematischen Gruppen zusammengefa $\beta$ t, das Zielpublikum reichte von jungen Müttern über Hebammen und Fürsorgerinnen bis zu Ärzten, Sozialhygienikern und Verwaltungsbeamten.

Den jungen Müttern wurde eine rationelle Schwangerschafts- und Wochenbetthygiene vorgestellt, Informationen über die kindliche Entwicklung sollten ihr Verständnis für die vom Arzt verordneten Pflege- und Ernährungshinweise wecken und eine Krankheitslehre ihnen ermöglichen, sowohl die Krankheiten als auch deren Ursachen zu erkennen und entsprechende Maßnahmen zu ergreifen. Den Ärzten stand mit der Abteilung über die Krankheiten des Säuglings eine Lehrmittelsammlung pathologisch-anatomischer Präparate und Moulagen zur Verfügung, und Sozialhygieniker und Verwaltungsbeamte konnten sich anhand von Kurven und Tabellen über den aktuellen Stand der Mortalitäts- und $\mathrm{Na}$ talitätsentwicklung informieren und in der Abteilung "Allgemeine Gesundheitspflege» die zu einer Verbesserung nötigen Veränderungen studieren. Die gesamte Ausstellung diente der Verdeutlichung von Zusammenhängen zwischen sozialer Lage, hygienischen Bedingungen, Pflegepraktiken und der Gesundheit des Säuglings. Obwohl das Museum einen guten systematischen Uberblick bot, genügte es den Ansprüchen Langsteins und Rotts an "Volksaufklärung» nicht. Es war «an den Ort gebannt (und) zu sehr von wis-

16 Langstein/Rott: Vorwort zum Atlas, 1918, S. 1. 
senschaftlichem Geiste durchtränkt, als daß es jene Anziehungskraft auf die Masse hätte, wie wir sie wünschen..... Das lebendige Bild mu $\beta$ wandern können und deshalb stelIten wir das Wesentliche durchweg in allgemein verständlicher Form zusammen und brachten es in Wanderausstellungen in weite Kreise unseres Volkes $\gg^{17}$.

Wichtiger Teil dieser Wanderausstellungs-Matorialien war ein sogenannter Atlas, d. h. eine Sammlung großer Schautafeln. In 100 Abbildungen sind alle wesentlichen Aspekte der Säuglings- und Kleinkindpflege behandelt. Den größten Bereich stellt mit 38 Titeln die hygienische Pflege des Kindes dar. Auf den Tafeln werden auch die traditionellen Praktiken dargestellt, die mit roten Balken als «verboten» durchkreuzt sind. Der Ernährung werden 20 Tafeln gewidmet, auf denen der Wert des Stillens und die hygienischen Anforderungen an die Flaschenernährung gezeigt wurden. Tafeln über Körperbau und Entwicklung, insbesondere das Wachstum des Kindes, sollten das Verständnis dafür wecken, da $\beta$ ein Säugling physiologisch anders funktioniert als ein Erwachsener und z.B. Uberfütterung seinen Organismus schädigt. Weitere 15 Titel dienten dem Erkennen von Krankheiten, dem Wissen um Zusammenhänge und konkreten Handlungsanweisungen im Krankheitsfall. Diese Tafeln sind besonders deutlich und anschaulich. Außerdem gibt es noch eine Abteilung mit 9 Tafeln zur Statistik der Säuglingssterblichkeit. Vorlagen für die Statistiktafeln finden sich in der Denkschrift «Deutschlands Spende für Säuglings- und Kleinkinderschutz» von 191618. Möglicherweise haben die Abbildungen des Atlas' wiederum als Vorlage für die 1930 erschienene und im Kaiserin Auguste Victoria-Haus erarbeitete «Ernährungsfibel für Mutter und Kind» gedient ${ }^{19}$.

Dem praktischen Anspruch werden zwei Tafeln besonders gerecht, auf denen die Schnittmuster für Säuglingsund Kleinkindkleidung abgedruckt sind. Sie waren von einer Fürsorgeschwester entworfen worden, die im ersten Beruf Schneiderin gewesen war und in ihren Entwürfen den medi-

\footnotetext{
17 ebda.

18 Für die übrigen Tafeln konnte ich keine entdecken. Auch die Namen der Künstler sind mir nicht bekannt.

19 Diesen Hinweis verdanke ich Frau Professor Ballowitz, die ihn im KAVH-Archiv, Mappe 7653 gefunden hat.
} 
zinisch festgestellten Bewegungsdrang der Kleinkinder berücksichtigte ${ }^{20}$.

In einem dem Atlas beigefügtem «Leitfaden»-Text werden zu jeder Tafel noch einige Informationen gegeben. Für Mütterabende oder den Unterricht an Volksschulen waren Pläne ausgearbeitet, welche Tafeln in welchem Zusammenhang gezeigt werden sollten.

Die $35 \times 50 \mathrm{~cm}$ großen Tafeln waren für die Wanderausstellungen auf Pappe aufgezogen und wurden verliehen. Einige mehr oder weniger gelungene Exemplare demonstrieren die pädagogisch-didaktischen Prinzipien der Darstellung.

Eine Tafel mit dem Titel Sterblichkeit nach Ernährungsweise und wirtschaftlichen Verhältnissen thematisiert die beiden wichtigsten Ursachen für Säuglingssterblichkeit: Von Säuglingen, die nicht gestillt wurden, starben viermal mehr als von den Brustkindern. Die soziale und wirtschaftliche Lage wirkten auf nichtgestillte Säuglinge ungleich stärker. Dieser Tatbestand wird durch zwei gegenübergestellte Bilder verdeutlicht, auf denen jeweils die Bedingungen - Stillen oder künstliche Ernährung - und die Konsequenzen gezeigt werden. Die unterschiedlich hohe Sterblichkeit wird durch verschieden hohe Grabmonumente deutlich gemacht. Für die Aussage dieser Tafel ist der Text nicht unbedingt nötig (Abb. 2).

Die allgemeine Erziehung zur Hygiene ist den Autoren des Atlas' als Grundlage der Säuglingspflege ein wichtiges Anliegen. Diesem Erziehungsziel dient besonders eine Tafel, die das Feindbild der Erreger in Form von Pilzen und Bakterien illustriert (Abb. 3). Sie werden in einer Form dargestellt, die aus der Alltagswelt verständlich ist: Schimmelpilze auf dem Brot, Eiter in einer Wunde sind bekannte und sichtbare Phänomene. Per Mikroskap wird eine genaue Darstellung und damit die Sichtbarmachung des Unsichtbaren vorgenommen, um eine Vorstellung für die Gefahren zu wecken, die in weniger sichtbaren Erregern lauern.

Abbildung 4 zeigt auf beeindruckende Weise die Mundreinigung und die Folgen durch Schemazeichnungen der Verletzungen der Mundschleimhaut.

Mehrere Tafeln beschäftigen sich mit Erkrankungen, sowohl mit ihrem Erkennen - wie die in Abbildung 5 darge-

${ }^{20}$ Für diesen Hinweis danke ich Schwester Hedwig Wegmann. 
stellte Tafel, die die verschiedenen Darmentleerungen zeigt als auch mit gezielten Gegenmaßnahmen. Abbildung 6 zeigt ein Kind, das Verdauungsbeschwerden hat und sich deutlich unwohl fühlt. Die gut informierte Mutter gie $\beta$ t die Milch weg, auf dem Tisch steht schon der Tee mit Saccharin, der als einzige Diät erlaubt ist, und eine hilfsbereite Nachbarin

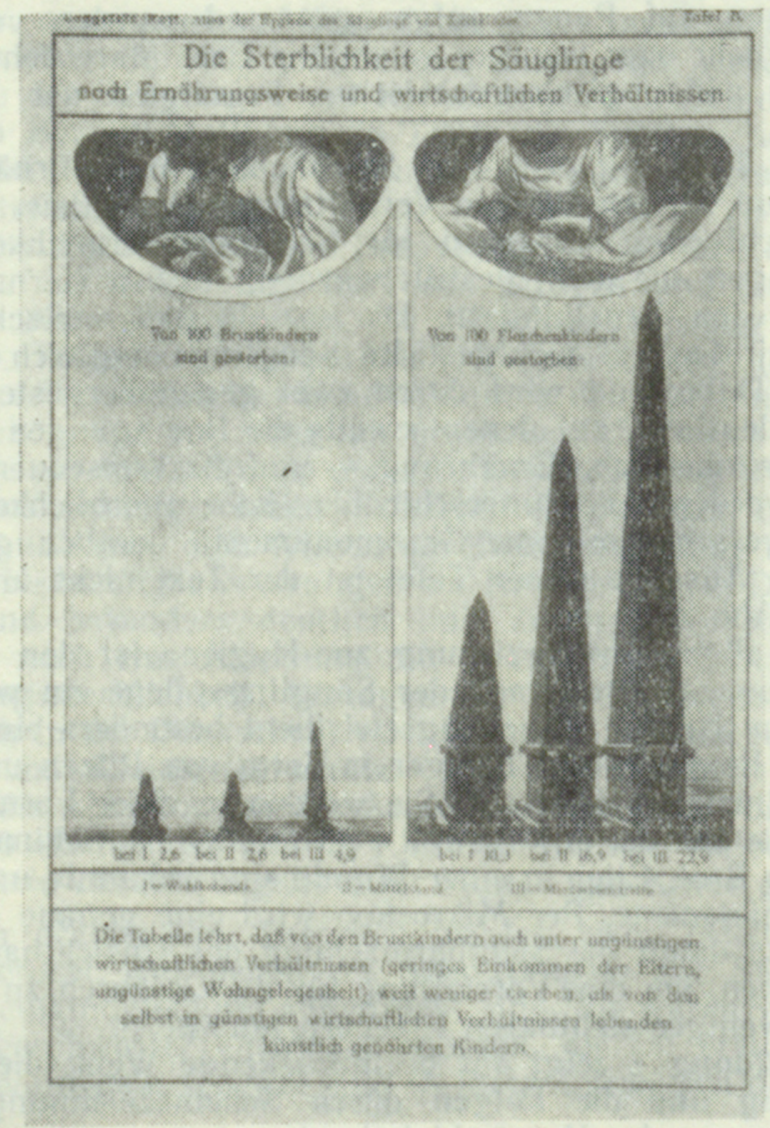

Abb. 2: Sterblichkeit nach Ernährungsweise und wirtschaftlichen Verhältnissen verdeutlicht den Vorteil des Stillens, Bei den nicht-gestillten Kinder gab es mehr als viermal soviel Todesfälle, insbesondere wenn sie in Armut lebten 


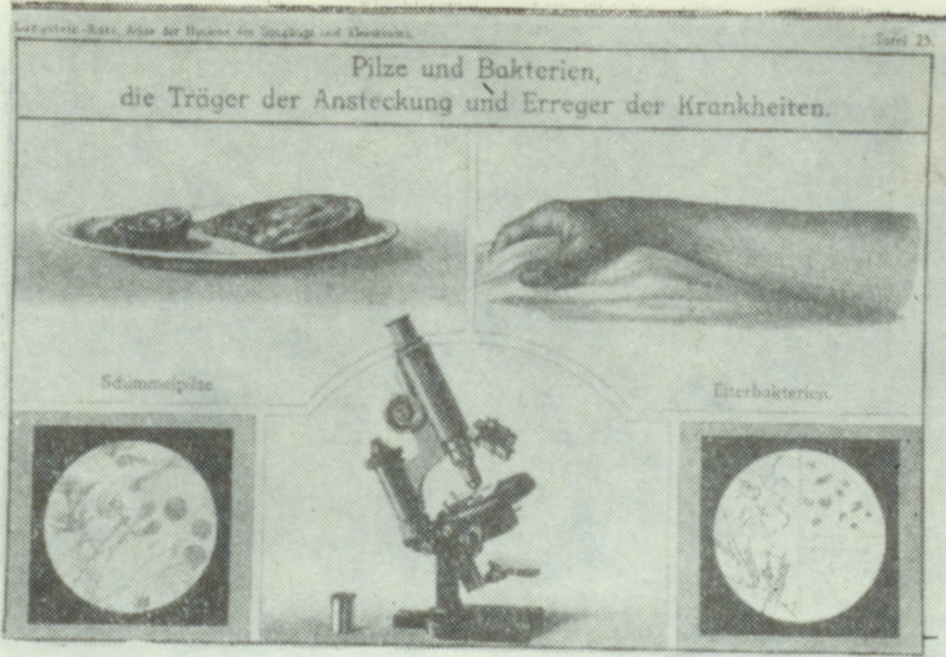

Abb. 3: Pilze und Bakterien. Die Tafel zeigt, wo im täglichen Leben Krankheitserreger auftreten

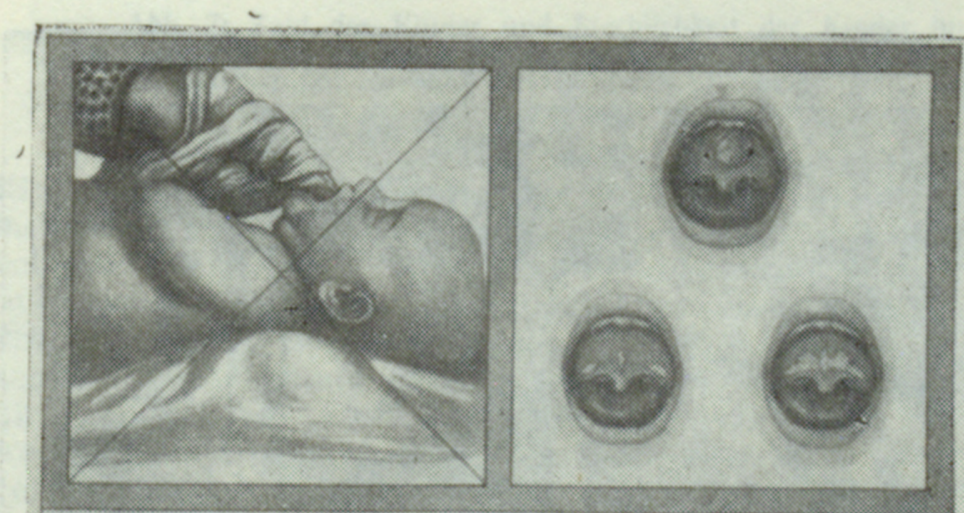

Die Mundreinigung des Säuglings ist stréng verboten, denn die Mundschleimhaut ist zart und leicht verletzlick. Bösartige Geschwüre in Munde können die Folge sein.

Abb. 4: Mundreinigung und die Folgen illustriert in einer Schemazeichnung die Verletzung der Mundschleimhaut 


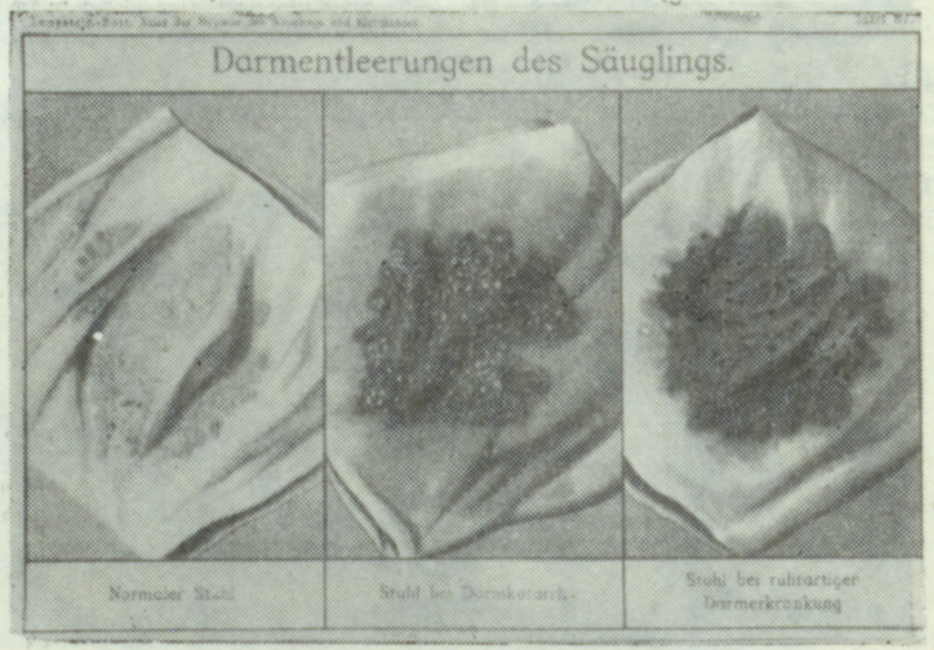

Abb. 5: Darmentleerungen des Säuglings. Mütter sollten auf den Stublgang des Säuglings achten, denn er ist ein sicheres Zeichen für sain Wohlbefinden

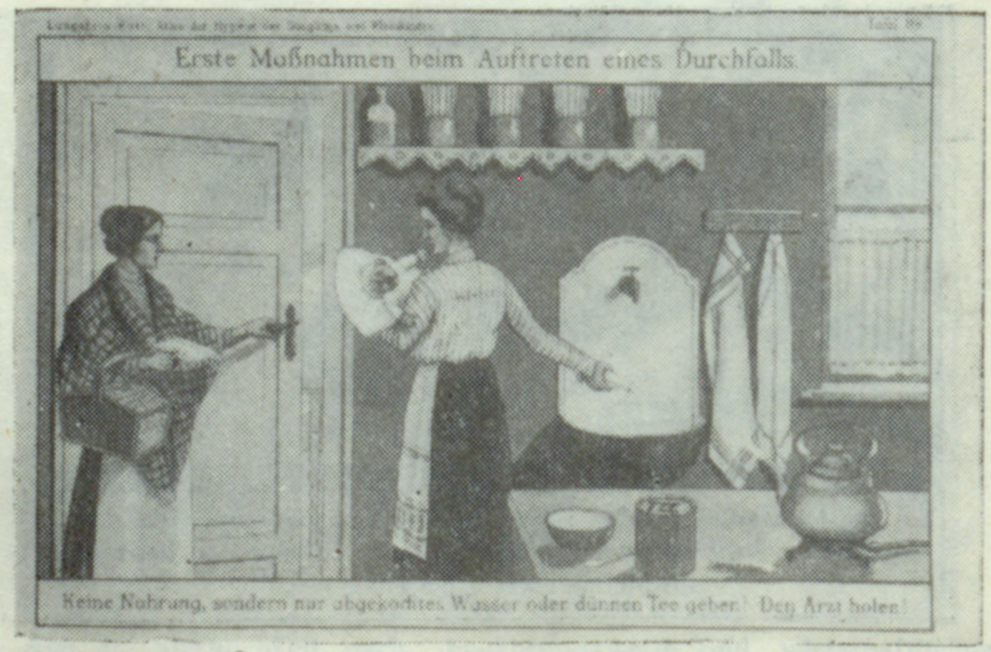

Abb. 6: Erste Maßnahmen beim Auftreten eines Durchfalls zeigt in einem Szenarium richtiges Verhalten 
holt den Arzt. Eine weitere Tafel zeigt, wie man dem vorbeugen kann, indem die Milch mit einfachen Mitteln unterm Wasserstrahl kühl gehalten wird.

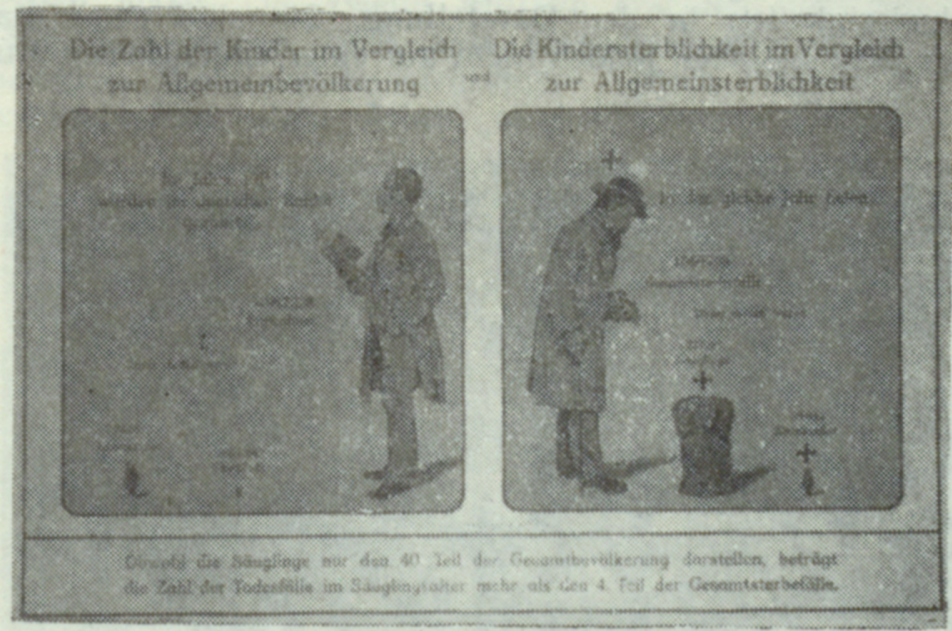

Abb. 7: Zahl der Kinder und Sterblichkeit der Kinder im Vergleich zur Allgemeinbevölkerung ist ein Beispiel für eine ungünstige Darstellungsform

Für weniger gelungen halte ich die Tafel über Zahl der Kinder und Sterblichkeit der Kinder im Vergleich zur Allgemeinbevölkerung (Abb. 7). Hier schlägt das sonst so sinnvoll verwandte Darstellungsprinzip, abstrakte Verhältnisse in konkreten Symbolen zu verdeutlichen, in sein Gegenteil um. Die Zahlen über den Anteil der Säuglinge an der Gesamtbevölkerung und die Höhe der Säuglingssterblichkeit im Vergleich zur Sterblichkeit der übrigen Altersgruppen werden in Form von Personen verschiedener Größe dargestellt. Das wird problematisch, weil man hier das natürliche Größenverhältnis zwischen Erwachsenen und Kleinkindern assoziiert. Entsprechend ist die Säuglingswiege auf dem linken Bild sehr klein, auf dem rechten Bild dagegen, das eigentlich die überdimensionierte Sterblichkeit der Säuglinge zeigen soll, steht die Größe der Wiege ungefähr im realen Verhältnis zum Erwachsenen. Genau das ist jedoch nicht die beabsichtigte Aussage der Tafel! 
Auch auf der Tafel zum internationalen Vergleich der Säuglingssterblichkeit (Abb. 8) sind keine Werte auf einer Skala eingetragen, sondern Gegenstände: Grabkreuze als Bedeutungsträger sind zwar verständlich, machen aber Schwierigkeiten in der Darstellung. Sie sind zwar maßstabsgetreu, stehen jedoch nicht auf einer einheitlichen Null-

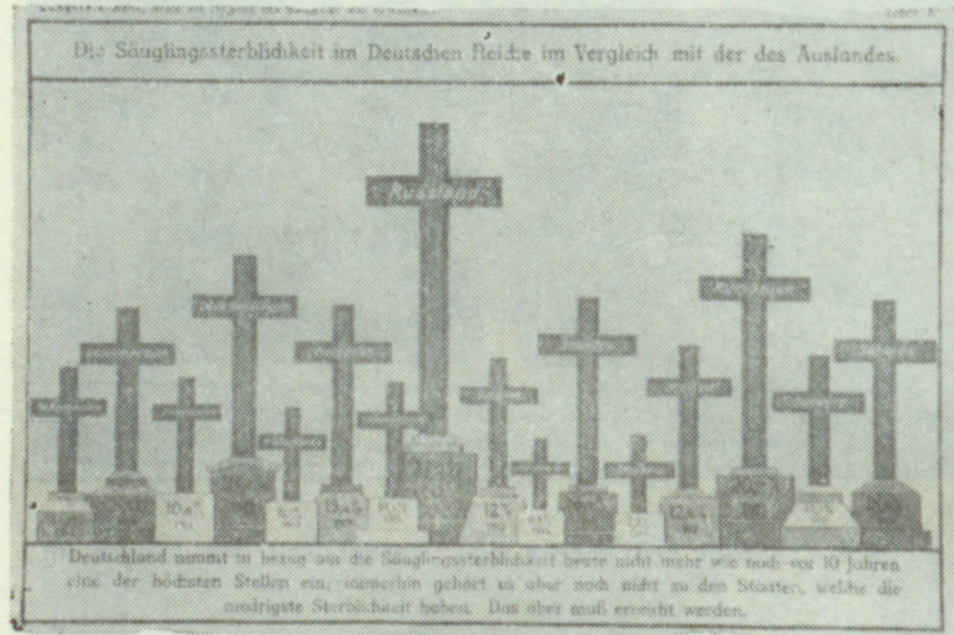

Abb. 8: Internationaler Vergleich der Säuglingssterblichkeit verfälscht die Aussagen, weil die Symbole nicht auf einer Ebene stehn

Linie, sondern nach Größe hintereinander, was der Anordnung auf einer Zick-Zack-Linie entspricht. Dadurch, da $\beta$ die größten hinten stehen, ragen sie noch weiter gegen den Hintergrund hinaus. Zudem befindet sich jedes Kreuz auf einem Sockel, der bei großen Kreuzen natürlich gro $\beta$, bei kleinen klein ist und so jede Aussage überzeichnet. Außerdem stammen die Werte aus verschiedenen Jahren.

Eine Tafel ist der je nach Jahreszeit unterschiedlichen Säuglingssterblichkeit gewidmet. Sie ist ästhetisch sehr ansprechend, aber die Gründe für eine hohe Säuglingssterblichkeit gerade im Sommer werden in der Abbildung nicht deutlich. Anstatt an einem kühlen See unter Bäumen - womit die Tafel Sommer symbolisiert - lebten und litten die 
Im Sommer verstorbenen Säuglinge in aufgeheizten, schlecht lüftbaren Großstadtwohnungen.

Die Nachfrage war so gro $\beta$, da $\beta$ die erste Auflage des Atlas' schon fast allein durch die Subskribenten 1920 vergriffen war $^{21}$. Die zweite Auflage erschien in Höhe von 1.500 Exemplaren 1922. Das Organisationsamt hatte an mehrere Wohlfahrtsteinrichtungen einen Spendenaufruf gerichtet, um den Atlas an Behörden, Organisatio-

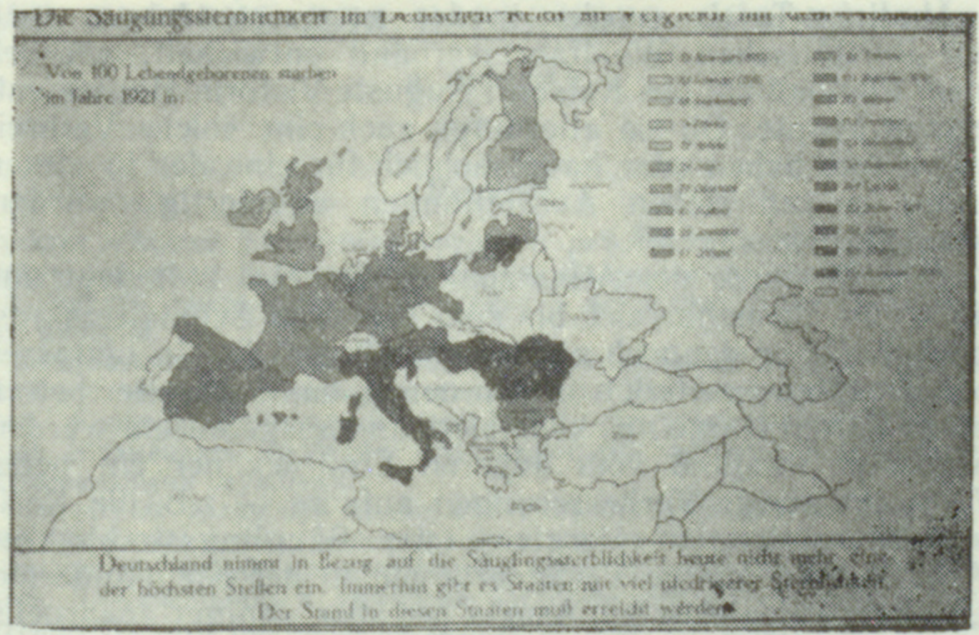

Abb. 9: Internationaler Vergleich der Säuglingssterblichkeit aus der Auflage von 1926/27 stellt die entsprechende Höhe in unterschiedlicher Färbung ider Länder auf der Landkarte dar

nen, Verbände, Anstalten und Einzelpersonen verbilligt abgeben zu können. Das Deutsche Rote Kreuz, die Deutsche Kinderhilfe, Deutschlands Spende für Säuglings- und Kleinkinderschutz sowie der Deutsche Caritasverband hatten der Bitte entsprochen ${ }^{22}$. Das Gros der verbilligten Exemplare (348) ging an staatliche und kommunale Behörden, 120 At-

${ }^{21}$ Bericht des Kaiserin Auguste Victoria-Hauses, Reichsanstalt zur Bekämpfung der Säuglings- und Kleinkindersterblichkeit, erstattet von Prof. Dr. L. Langstein vom 1. April 1918 bis, 31. März 1919, S. 25, und vom 1. April 1920 bis 31. März 1921, S. 15.

22 Bericht des Kaiserin Auguste Victoria-Hauses, Reichsanstalt zur Bekämpfung der Säuglings- und Kleinkindersterblickeit, erstattet von Prof. Dr. L. Langstein vom 1. April 1921 bis 31. März 1922, S. 29. 
lanten an Organisationen der Mutter-, Säuglings- und Kleinkinderfürsorge, 103 an Krankenhäuser und wissenschaftliche Institute, 76 an Schulen, 42 an Ärzte, 23 an Fürsorgerinnen, Säuglings- und Krankenpflegerinnen und 38 an Drogerien ${ }^{23}$. Die Drogerien wurden vermutlich mit dem Atlas bedacht, weil sich ratsuchende Mütter dorthin wandten, wenn sie irgendein Medikament oder Hausmittel gegen das Unwohlsein ihres Kindes suchten, und dann mit den Tafeln über richtige Pflege informiert wurden ${ }^{24}$.

Ähnliche Tafeln wurden in den zwanziger Jahren auch vom Paritätischen Wohlfahrtsverband entwickelt, wie ich einem Photoalbum des Kaiserin Auguste Victoria-Hauses entnehmen konnte. Ob sie allerdings auch eine solche Verbreitung erlebt haben oder hauptsächlich für eine der in diesen Jahren so zahlreichen Ausstellungen angefertigt wurden, kann ich nicht sagen ${ }^{25}$.

1926/27 wurde der Atlas zum dritten Mal aufgelegt und dabei sein Themenbereich bis zum Ende des Schulalters ausgedehnt. Da er dennoch nicht mehr als 100 Tafeln umfassen sollte, mußten zusätzlich zu den neuen, das Schulalter betreffenden Tafeln einige neu konzipiert und ausgetauscht werden. Insgesamt entstand vom äußeren Einđruck her ein neues Werk, das einige Verbesserungen aufwies - z.B. die Karte zum internationalen Vergleich der Säuglingssterblichkeit (Abb. 9), oder einige Veränderungen in der Kleinkindermode - vom künstlerischen Aspekt her aber weniger anspruchsvoll war. Die Darstellungen wirken flacher, schematischer. Dennoch war 1929 auch diese Auflage bis auf einen kleinen Rest vergriffen ${ }^{26}$.

Zum 80 jährigen Jubiläum des Kaiserin Auguste VictoriaHauses wurde der Atlas in verkleinertem Format wiederaufgelegt. Die Herausgeber bezeichnen ihn im Vorwort als herausragendes Beispiel für die damaligen Bemühungen um

23 Bericht des Kaiserin Auguste Victoria-Hauses, Reichsanstalt zur Bekämpfung der Säuglings- und Kleinkindersterblichkeit, erstattet von Prof. Dr. L. Langstein vom 1. April 1922 bis 31. März 1923, S. 31.

24 Zur historischen Nähe von Drogerien und Apotheken vgl. Münch, Ragnhild: Zur Berufsstruktur des Berliner Gesundheitswesens am Ende des 18. Jahrhunderts, in: Berlin-Forschungen I, Hrsg. W. Ribbe, Berlin 1986, S. $198-218$, hier S. $210-216$.

25 Information von Frau Ballowitz.

26 Bericht des Kaiserin Auguste Victoria-Hauses, Reichsanstalt zur Bekämpfung der Säuglings- und Kleinkindersterblichkeit, erstattet von Prof. Dr. L. Langstein vom 1. April 1927 bis 31. März 1929, S. 75. 
Volksaufklärung und empfehlen ihn «für sinnvolle Aufklärungs- und Fürsorgetätigkeit in Entwicklungsländern». Ich halte ihn eher für eine wichtige Dokumentation der gesundheitspolitischen und sozialhygienischen Bestrebungen sowie ihrer pädagogischen Konzepte und deren ästhetischer Umsetzung, die im Paul-Stradin-Museum besser aufgehoben ist!

\title{
АТЛАС ПО ГИГИЕНЕ ГРУДНОГО РЕБЕНКА И ДЕТЕИ РАННЕГО ВОЗРАСТА КАҚ ПОСОБИЕ ДЛЯ НАРОДНОГО ПРОСВЕЩЕНИЯ
}

\author{
3. ШTEKЕЛ \\ Берлин
}

\section{Резюме}

Издание «Атласа по гигиене грудного ребенка и детей раннего возраста» относится к мероприятиям, направленным на борьбу с высокой детской смертностью в Германии в последние годы существования империи.

Взаимосвязь социального положения и высокой детской смертности была очевидна и законодательно взята под контроль государства лишь в период Веймарской республики. До этого медицинская помощь оказывалась свободно практикующими врачами и некоторыми коммунальными службами.

Атлас был издан при поддержке Главного управления Отечественного женского союза, директора учреждения императрицы АвгустыВиктории по борьбе со смертностью грудных младенцев в Германии проф. Л. Лангштейна и директора организационного управления императрищы Августы-Виктории по защите прудных младенцевдокт. Ф. Рота.

В 1906 г. под покровительством императрицы был создан «Германский комитет по организации образцового учреждения по борьбе со смертностью грудных младенцев», открытый в 1909 г. На него возлагались задачи по исследованию причин детской смертности и методов ее профилактики. До 1911 г. это учреждение возглавлял докт. А. Келлер, из клиники Черни в Бреславле, а затем докт. Лангштейн, работавший до этого в детской клинике Шарите в Берлине. Все эти события нашли свое отражение на страницах атласа.

Иллюстрации и таблицы, помещенные в атласе, давали основные сведения по анатомии, физиологии и гигиене грудных младенцев, o возбудителях, путях и способах распространения инфекционных бо лезней, а также ряд других санитарно-просветительных рекомендаций

В 1927 г. в ознаменование 80-летия со дня рождения императрицы Августы-Виктории атлас был переиздан в уменьшенном объеме.

Dr. med. Sigrid Stöckel

Abteilung für Geschichte der Medizin,

Medizinische Hochschule,

Postfach 610180

3000 Hannover 61,

Bundesrepublik Deutschland 